\title{
Causal mechanisms of a healthy lifestyle intervention for patients with musculoskeletal pain who are overweight or obese
}

Williams $A^{1,2,3}$, Lee $H^{1,3,4,5}$, Kamper $S J^{3,6}$, O’Brien $\mathrm{KM}^{1,2,3}$, Wiggers $\mathrm{J}^{1,2}$, Wolfenden $\mathrm{L}^{1,2}$, Yoong $\mathrm{SL}^{1,2}$, Hodder $\mathrm{RK}^{1,2,3}$, Robson $\mathrm{EK}^{1,2,3}$, Haskins $\mathrm{R}^{7}$, McAuley $\mathrm{JH}^{3,4}$, Williams $\mathrm{CM}^{1,2,3}$

${ }^{1}$ School of Medicine and Public Health, Hunter Medical Research Institute, University of Newcastle, Newcastle NSW, 2308, Australia

${ }^{2}$ Hunter New England Population Health, Locked Bag 10, Wallsend NSW, 2287, Australia.

${ }^{3}$ Centre for Pain, Health and Lifestyle, NSW, Australia

${ }^{4}$ Neuroscience Research Australia (NeuRA), PO Box 1170, Randwick NSW, 2031, Australia

${ }^{5}$ Centre for Statistics in Medicine, Nuffield Department of Orthopaedics Rheumatology and Musculoskeletal Sciences, University of Oxford, Oxford, UK

${ }^{6}$ School of Public Health, University of Sydney, Lvl 10, King George V Building, Camperdown NSW, 2050, Australia

${ }^{7}$ Outpatient Services, John Hunter Hospital, Hunter New England Local Health District, Locked Bag 1, New Lambton NSW, 2305, Australia

Corresponding author: Amanda Williams, Amanda.j.williams@hnehealth.nsw.gov.au 


\section{Abstract}

We assessed the causal mechanisms of a healthy lifestyle intervention for patients with chronic low back pain and knee osteoarthritis $(O A)$, who are overweight or obese. We conducted causal mediation analyses of aggregated data from two RCTs; which included 160 patients with chronic low back pain, and 120 patients with knee OA. Participants were randomised via one central randomisation schedule, to the intervention, or usual care. The intervention consisted of brief advice and referral to a 6-month telephone-based healthy lifestyle coaching service. Participants in the back pain trial were also offered a single physiotherapy consultation. The hypothesised primary mediator was self-reported weight, and alternative mediators were diet, physical activity, and pain beliefs. Outcomes were pain intensity, disability, and quality of life (QoL). Data were analysed using causal mediation analyses with sensitivity analyses for sequential ignorability. All mediation models were specified a priori. The intervention had no effect on pain intensity, disability or physical QoL. The intervention significantly improved mental QoL, however, the intervention effect was not channelled via the selected mediators. The intervention did not reduce weight, or the alternative mediators (diet, physical activity, pain beliefs), and these mediators were not associated with the outcomes (with one exception; poor diet was associated with lower mental QoL). The sensitivity analyses showed that our estimates were stable across all possible levels of residual confounding. Our findings show that the intervention did not cause a meaningful change in the hypothesised mediators, and these mediators were not associated with patient outcomes. 


\section{Background}

Low back pain and knee osteoarthritis (OA) are common musculoskeletal conditions responsible for a significant global burden. ${ }^{1}$ In the latest Global Burden of Disease Study (2016), low back pain ranked $1^{\text {st }}$ and $O A$, for which knee $O A$ is the highest contributor, ranked $12^{\text {th }}$ among all causes of years lived with disability. ${ }^{1}$ Consequently, these conditions cause substantial economic strain. For example, the total annual cost to Australian society was estimated at $\$ 9.2$ billion $(2001)^{2}$ for low back pain and $\$ 23.1$ billion $^{3}$ (2008) for OA.

A number of factors potentially affect the course of low back pain and knee OA. Among those commonly reported are lifestyle risk factors and erroneous pain beliefs. For example, meta-analyses have shown that being overweight or obese is associated with the persistence of low back pain ${ }^{4,5}$ and is an adverse prognostic factor for knee OA. ${ }^{6,7}$ Given their influence on weight gain, lifestyle risk factors such as poor diet and physical inactivity are also likely to indirectly influence the course of low back pain and knee OA, via weight status. ${ }^{8,9}$ Independently, physical inactivity is directly associated with the persistence of low back pain ${ }^{10}$ and poorer physical function in people with knee OA. ${ }^{11}$ In addition, erroneous pain beliefs are known to adversely influence outcomes from low back pain and knee OA resulting in delayed recovery and higher disability. ${ }^{12,13}$

Targeting lifestyle risk factors and erroneous pain beliefs are considered important aspects of treatment programs for managing chronic low back pain and knee OA. ${ }^{14,15}$ We conducted two randomised controlled trials (RCTs) of complex interventions targeting weight, diet, physical activity and pain beliefs, aiming to reduce pain intensity in patients with chronic low back pain ${ }^{16}$, and patients with knee OA, ${ }^{17}$ who are overweight or obese. Standard analyses of RCTs estimate whether an intervention is effective or not. ${ }^{18,19}$ However, these analyses cannot provide explanations for how an intervention works, or why they do not. ${ }^{20}$ To do so, causal mediation analysis of RCTs can be used to determine the extent to which a selected treatment target (mediator) channels the effect of the treatment onto the primary outcome. ${ }^{20}$ Such analyses are important to generate evidence to refine interventions, with the aim of improving their effectiveness. For example, treatment components that target effective mediators can be prioritised and strengthened in future iterations of that intervention. Conversely, mediation analyses can also explain why an intervention is ineffective. That is, by determining whether it was the intervention that failed to influence mediators, or whether the mediators were not associated with outcomes, or both. ${ }^{18,21}$

The underlying mechanisms of lifestyle interventions for patients with chronic low back pain and knee OA have rarely been tested. ${ }^{18}$ To our knowledge, only one study of a lifestyle 
intervention in a similar population group has investigated treatment mechanisms. Foy et al. found that in adults with knee pain and diabetes, who were overweight or obese, a reduction in weight mediated the intervention effect on disability. ${ }^{22}$ Given the paucity of research, the objective of this study was to test the underlying causal mechanisms of a healthy lifestyle intervention for patients with chronic low back pain or knee OA, who are overweight or obese.

\section{Methods}

\section{Study design and participants}

We conducted causal mediation analyses on aggregated data from two, two-arm RCTs, both part of a cohort multiple RCT. ${ }^{23,24}$ Full details of the methods of each trial are outlined in Williams et al. ${ }^{16,23}$ (ACTRN12615000478516) and O'Brien et al. ${ }^{17,24}$ (ACTRN12615000490572). Briefly, all patients were recruited from a waiting list for outpatient consultation with an orthopaedic specialist at the John Hunter Hospital, New South Wales (NSW), Australia. One RCT involved 160 patients with chronic non-specific low back pain, ${ }^{23}$ and the other, 120 patients with knee OA. ${ }^{24}$ All patients across both trials had a body mass index of $\geq 27 \mathrm{~kg} / \mathrm{m}^{2}$ and $<40 \mathrm{~kg} / \mathrm{m}^{2}$ based on self-reported weight and height. Participants were randomised to both trials via one central randomisation schedule, to receive a healthy lifestyle intervention (intervention group), or remain in the cohort follow up (usual care control group), in a 1:1 ratio. The randomisation schedule was generated a priori by an independent investigator using SAS 9.3 through the SURVEYSELECT procedure. The pre-specified analysis plan for the current study is outlined in Lee et al. $2017 .^{25}$

\section{Intervention}

In both trials, participants allocated to the intervention group received brief telephone advice provided by trained telephone interviewers immediately after baseline assessment and randomisation. This advice included information about the potential benefits of weight loss and physical activity for low back pain or knee OA. Participants were then referred to the NSW Get Healthy Service (GHS) (www.gethealthynsw.com.au). ${ }^{26}$ The GHS is a free public health telephone-based service provided by the NSW Government to support adults to make sustained lifestyle improvements including diet, physical activity, and achieving or maintaining a healthy weight. ${ }^{26}$ All GHS health coaches were trained in evidence-based advice for chronic low back pain and knee OA. This training involved a 2-hour interactive workshop and information resources to guide advice for study participants. 
Participants in the chronic low back pain trial were also offered a clinical consultation with the study physiotherapist. The consultation involved a clinical assessment, patient education to correct erroneous pain beliefs and behaviour change techniques to facilitate healthy lifestyle habits and weight management, informed by Self Determination Theory. ${ }^{27}$ Although pain beliefs were not directly targeted in the knee OA trial, we hypothesised that promotion of physical activity by the GHS service could change pain beliefs (e.g. that pain does not need to be a barrier to a physically active lifestyle).

\section{Control}

Participants allocated to the control group continued on the usual care pathway (i.e. remained on the waiting list to have an orthopaedic consultation and could progress to consultation if scheduled) and took part in data collection during the study period. No other active intervention was provided as part of the study, however; no restrictions were placed upon the use of other health services during the study period. Control participants were informed that a new clinical service would be available in approximately 6 months involving clinical assessment and support from other services for their back pain or knee OA should they need it. No other details about the new service, or that other patients had started this service were disclosed.

\section{Measures}

\section{Mediators}

The selected primary mediator was self-reported weight, in kilograms. Alternative mediators were: physical activity measured using the Active Australia Survey, ${ }^{28}$ which has moderate reliability (Cohen's Kappa $=0.52)^{29}$ and good face and criterion validity; ${ }^{30}$ dietary intake measured using a short food frequency questionnaire, ${ }^{31}$ which has moderate reliability (Weighted Kappa range $=0.37$ to 0.85$)^{32,33}$ and criterion validity; ${ }^{33}$ and pain related attitudes and beliefs measured using the Survey of Pain Attitudes One-item Questionnaire, which is strongly associated with the parent questionnaire that has acceptable levels of reliability and validity. ${ }^{34,35}$

\section{Outcomes}

The primary outcomes were average self-reported pain intensity over the previous 7-days, measured using an 11-point pain Numeric Rating Scale ( $0=$ no pain, $10=$ worst possible pain) ${ }^{36}$ self-reported disability measured using the 24 -item Roland-Morris Disability Questionnaire (RMDQ) in participants with chronic low back pain, ${ }^{37}$ and the Western Ontario and McMaster Universities Osteoarthritis Index (WOMAC) ${ }^{38}$ in participants with knee OA; 
and physical and mental quality of life (QoL) measured using the Short Form Health Survey 12 V.2. ${ }^{39}$ All outcomes are widely used and validated measures for these populations. ${ }^{36-40}$

\section{Potential confounders}

We identified potential confounders of the mediator-outcome effects based on theorised causal effects on the mediator and outcome variables. The selected confounders were: duration of pain (years since onset), pain intensity, disability and QoL, all measured at baseline.

\section{Data collection}

Participant characteristics, primary and alternative mediators, outcomes and potential confounders were measured at baseline prior to random allocation by telephone interview. The primary mediator (self-reported weight) was measured 6 months after randomisation. The alternative mediators (diet, physical activity, pain beliefs) were measured 6 weeks after randomisation. The different timing of the measurement of the primary and alternative mediators was planned a priori to facilitate analysis via multiple mediator models (if appropriate), as per the pre-specified analysis plan outlined in Lee et al. $2017 .{ }^{25}$ The outcomes (pain intensity, disability, and QoL) were measured 6 months after randomisation. All mediators and outcomes were collected by a questionnaire completed via telephone by trained telephone interviewers blind to group allocation or mailed in the post as per participant preference.

\section{Statistical analysis}

We used causal mediation analyses to analyse the data following the pre-specified analysis plan outlined in Figure 2 of Lee et al. $2017 .{ }^{25}$ We conducted all analyses in R (The R Foundation for Statistical Computing) using the "mediation" package. ${ }^{41}$

We constructed independent single mediator models for each hypothesised mediator (weight, diet, physical activity and pain beliefs) for each outcome (pain intensity, disability, physical QoL and mental QoL). Directed acyclic graphs for each model are shown in Figure 1. 
Figure 1. Directed acyclic graph representing a single mediator model where the intervention exerts its effect on the outcome (i.e. pain intensity/disability/QoL - physical/QoL - mental), via an indirect path (blue lines) through the mediator (i.e. weight/diet/physical activity/pain beliefs) and via a direct path (green line). Red lines represent possible effects that could induce confounding for indirect and direct effects. We assumed independence between all four mediators. Abbreviations: $\mathrm{QoL}=$ Quality of Life

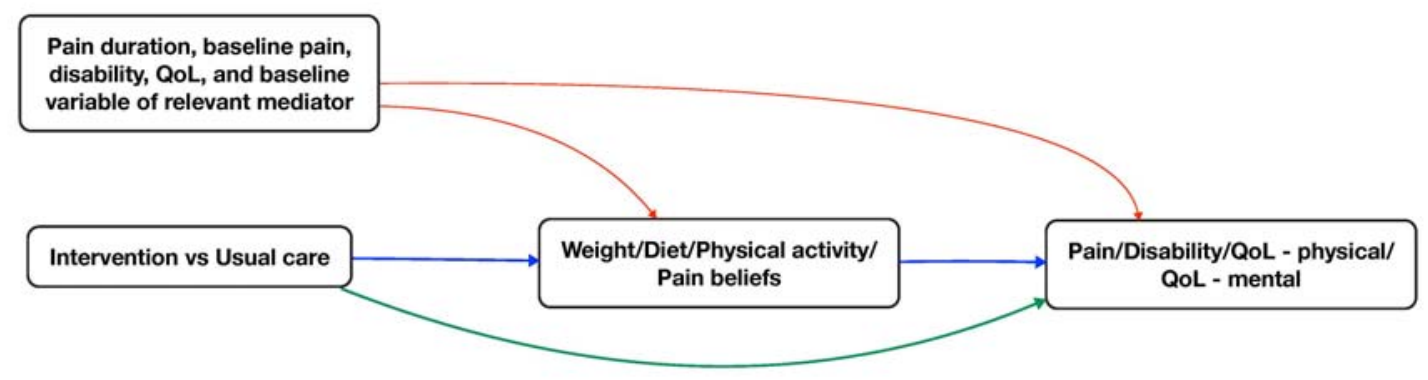

We assumed that the intervention-mediator and intervention-outcome paths were not confounded due to random allocation of patients to intervention and control groups. However, as the mediator cannot be randomised, the mediator-outcome path is likely to be confounded. Therefore, we included theorised potential confounders (duration of pain, baseline pain intensity, disability and QoL) in the outcome regression models as covariates.

For each model, we estimated the average total effect (ATE), average causal mediation effect $(A C M E)$, average direct effect (ADE), and the proportion mediated. The ACME is the intervention effect on the outcome via the mediator; $A D E$ is the intervention effect that is not channelled via the selected mediator; and ATE is the sum of ACME and ADE (the entire intervention effect). The proportion mediated is the fraction of ATE that is explained by ACME.

For each single mediator model, we fit two regression models: the mediator model and the outcome model. The mediator model was constructed with treatment allocation as the independent variable, and the mediator as the dependent variable. The outcome model was constructed with treatment allocation and the mediator as independent variables, the outcome as the dependent variable, and baseline measures of the mediator and the set of theorised potential confounders of the mediator-outcome path as covariates. ${ }^{42}$ We also included an interaction term (treatment allocation $\mathrm{X}$ mediator) in the outcome model to allow for a treatment-mediator interaction effect on the outcome. We used the mediate function to compute ATE, ACME, and ADE. 
We planned to present the aggregate data from both trials as per our pre-specified protocol. ${ }^{25}$ However, given that there were some differences between the two trials, namely the clinical populations (chronic low back pain and knee $O A$ ) and the additional physiotherapy consultation exclusively delivered in the back pain trial, it seemed plausible that effects could have been moderated by trial assignment. To determine whether this was the case, we used moderated causal mediation analysis to estimate both trial-specific effects, and average effects across both trials. We decided to interpret trial-specific effects rather than averaged effects if the ACME and ADE were conditional on trial assignment.

Our mediation models were not protected against residual confounding (i.e. due to unmeasured confounders) of the mediator-outcome path. Therefore, we explored how much residual confounding would explain away the indirect effect, by using sensitivity analyses. ${ }^{20}$ The level of residual confounding is represented by the correlation between the residuals (error terms) from the mediator and outcome models, denoted $\rho$ (rho). We used the medsens function to explore how varying levels of $\rho$ (between the extremes of -1 and +1 ) influenced the ACME. The output provides the value of $\rho$ at which the point estimate and $\mathrm{Cls}$ of the ACME includes 0 (no mediating effect). From this, we determined how strong the effect of unmeasured confounding would need to be to invalidate the estimated ACME.

\section{Deviations from the pre-specified analysis plan}

We made three deviations from the pre-specified analysis plan. First, the primary mediator, weight, was self-reported rather than objectively measured, this decision was made due to the availability of data. Second, we transformed the diet measure and the physical activity measure, from an ordinal and continuous scale respectively, to a binary scale to benchmark the measures against Australian Guidelines. ${ }^{43,44}$ A score of ' 1 ' indicates meeting the guidelines (i.e. diet: 2 or more serves of fruit and 5 or more serves of vegetables per day; physical activity: participation in $\geq 150$ minutes of moderate to vigorous physical activity per week) and ' 0 ' indicates not meeting these guidelines. Third, we harmonised measures of disability (RMDQ in participants with chronic low back pain, ${ }^{37}$ and the WOMAC ${ }^{38}$ in participants with knee $O A$ ) to facilitate the interpretation of aggregate data from the two trials. We computed standardised scores for disability using the method of Van Cleave et al. 2011. ${ }^{45}$ These procedures are described in Text $S 1$ in the supplementary file.

\section{Results}

Trial assignment (chronic low back pain vs. knee OA trial) did not moderate the ACME nor ADE for all single mediator models. Thus, we present the aggregate ACME, ADE, and ATE from both trials. 


\section{Pain intensity}

The intervention had no effect on pain intensity. The intervention did not reduce the primary mediator (weight) and did not improve the alternative mediators (diet, physical activity, and pain beliefs). None of the mediators were associated with pain intensity (Table 1).

\section{Disability}

The intervention had no effect on disability. The intervention did not reduce the primary mediator (weight) and did not improve the alternative mediators (diet, physical activity, pain beliefs), and none of the mediators were associated with disability.

\section{Physical QoL}

The intervention had no effect on physical QoL. The intervention did not reduce the primary mediator (weight) and did not improve the alternative mediators (diet, physical activity, and pain beliefs), and none of the mediators were associated with physical QoL.

\section{Mental QoL}

The intervention significantly improved mental QoL, however, the intervention effect was not channelled via the selected mediators (Table 1). The intervention did not reduce the primary mediator (weight), and weight was not associated with mental QoL. The intervention did not improve the alternative mediators (diet, physical activity, and pain beliefs); and physical activity and pain beliefs were not associated with mental QoL. Diet was negatively associated with mental QoL (i.e. meeting the dietary guidelines for serves of fruits and vegetables per day was associated with poorer mental QoL).

\section{Sensitivity analyses}

The sensitivity analyses showed that our estimated ACME's were stable across all possible levels of residual confounding. The sensitivity plots for each model are reported in Figure S1 in the supplementary file.

\section{Multiple mediator models}

As per the pre-specified analysis plan, ${ }^{25}$ we did not conduct multiple mediator models because the intervention did not reduce weight (primary mediator). 
Table 1. Effect decomposition for each single mediator model

\begin{tabular}{|c|c|c|c|c|c|c|}
\hline Analysis & $\begin{array}{c}\text { Intervention-mediator } \\
\text { effect (path a) }\end{array}$ & $\begin{array}{l}\text { Mediator-outcome } \\
\text { effect (path b) }\end{array}$ & ATE & ADE & ACME & $\begin{array}{c}\text { Proportion mediated } \\
\text { (\%) }\end{array}$ \\
\hline \multicolumn{7}{|l|}{ Pain intensity } \\
\hline Diet & $0.79(0.50 \text { to } 1.25)^{\mathrm{a}}$ & $0.09(-1.41$ to 1.58$)$ & $0.11(-0.64$ to 0.82$)$ & $0.10(-0.65$ to 0.82$)$ & $0.01(-0.06$ to 0.08$)$ & $0.00(-0.71$ to 1.14$)$ \\
\hline Physical activity & $1.11(0.77 \text { to } 1.60)^{\mathrm{a}}$ & $-0.33(-1.62$ to 0.95$)$ & $0.13(-0.58$ to 0.85$)$ & $0.12(-0.58$ to 0.84$)$ & $0.01(-0.08$ to 0.12$)$ & $0.00(-1.20$ to 1.31$)$ \\
\hline Pain beliefs & $0.52(-0.71$ to 1.74$)$ & $0.05(-0.05$ to 0.14$)$ & $0.13(-0.62$ to 0.84$)$ & $0.12(-0.65$ to 0.82$)$ & $0.03(-0.05$ to 0.13$)$ & $0.01(-1.30$ to 2.18$)$ \\
\hline \multicolumn{7}{|l|}{ Disability } \\
\hline Weight & $1.50(-2.82$ to 5.81$)$ & $0.01(-0.02$ to 0.03$)$ & $0.12(-0.14$ to 0.37$)$ & $0.12(-0.13$ to 0.34$)$ & $0.00(-0.08$ to 0.10$)$ & $0.03(-2.23$ to 1.85$)$ \\
\hline Diet & $0.79(0.50 \text { to } 1.25)^{\mathrm{a}}$ & $0.13(-0.31$ to 0.56$)$ & $0.13(-0.10$ to 0.37$)$ & $0.15(-0.09$ to 0.38$)$ & $-0.02(-0.08$ to 0.02$)$ & $-0.05(-2.08$ to 1.25$)$ \\
\hline Physical activity & $1.11(0.77 \text { to } 1.60)^{\mathrm{a}}$ & $0.08(-0.34$ to 0.50$)$ & $0.14(-0.10$ to 0.38$)$ & $0.14(-0.10$ to 0.37$)$ & $0.00(-0.02$ to 0.03$)$ & $0.00(-0.61$ to 0.45$)$ \\
\hline Pain beliefs & $0.52(-0.71$ to 1.74$)$ & $0.01(-0.02$ to 0.04$)$ & $0.13(-0.10$ to 0.35$)$ & $0.12(-0.10$ to 0.34$)$ & $0.01(-0.02$ to 0.05$)$ & $0.03(-0.84$ to 1.01$)$ \\
\hline \multicolumn{7}{|l|}{ QoL - Physical } \\
\hline Diet & $0.79(0.50 \text { to } 1.25)^{\mathrm{a}}$ & $1.45(-2.99$ to 5.90$)$ & $-1.24(-3.78$ to 1.30$)$ & $-1.22(-3.79$ to 1.35$)$ & $-0.02(-3.79$ to 1.35$)$ & $0.01(-1.17$ to 1.06$)$ \\
\hline Physical activity & $1.11(0.77 \text { to } 1.60)^{\mathrm{a}}$ & $2.98(-1.42$ to 7.38$)$ & $-1.22(-3.95$ to 1.27$)$ & $-1.29(-4.09$ to 1.18$)$ & $0.07(-0.32$ to 0.55$)$ & $-0.01(-1.63$ to 1.62$)$ \\
\hline Pain beliefs & $0.52(-0.71$ to 1.74$)$ & $0.06(-0.25$ to 0.38$)$ & $-1.21(-4.12$ to 1.53$)$ & $-1.10(-3.85$ to 1.52$)$ & $-0.11(-0.60$ to 0.20$)$ & $0.05(-0.92$ to 0.84$)$ \\
\hline \multicolumn{7}{|l|}{ QoL - Mental } \\
\hline Weight & $1.50(-2.82$ to 5.81$)$ & $-0.11(-0.39$ to 0.17$)$ & $3.80(0.48 \text { to } 7.16)^{\star}$ & $3.90(0.70 \text { to } 7.02)^{*}$ & $-0.10(-1.29$ to 0.92$)$ & $-0.01(-0.96$ to 0.29$)$ \\
\hline Diet & $0.79(0.50 \text { to } 1.25)^{\mathrm{a}}$ & $-6.40(-12.06 \text { to }-0.74)^{*}$ & $4.73(1.40 \text { to } 8.10)^{\star}$ & $4.62(1.32 \text { to } 7.97)^{*}$ & $0.10(-0.53$ to 0.93$)$ & $0.03(-0.16$ to 0.29$)$ \\
\hline Physical activity & $1.11(0.77 \text { to } 1.60)^{\mathrm{a}}$ & $1.78(-4.01$ to 7.58$)$ & $4.70(1.34 \text { to } 8.00)^{\star}$ & $4.69(1.31 \text { to } 7.96)^{*}$ & $0.01(-0.37$ to 0.40$)$ & $0.00(-0.10$ to 0.11$)$ \\
\hline Pain beliefs & $0.52(-0.71$ to 1.74$)$ & $-0.23(-0.64$ to 0.18$)$ & $4.92(1.61 \text { to } 8.30)^{*}$ & $5.04(1.71 \text { to } 8.36)^{*}$ & $-0.12(-0.77$ to 0.30$)$ & $-0.02(-0.20$ to 0.06$)$ \\
\hline
\end{tabular}

\footnotetext{
Abbreviations: ATE = average total effect, $\mathrm{ADE}=$ average direct effect, $\mathrm{ACME}=$ ave

Adjusted coefficients $(\beta)$ with their $95 \%$ confidence intervals unless otherwise stated.

${ }^{\mathrm{a} B i n a r y}$ model presented as an odds ratio

${ }^{*} p=<0.05$
} 


\section{Discussion}

\section{Key findings}

Our findings showed that the healthy lifestyle intervention did not improve pain intensity, disability or physical QoL in patients with chronic low back pain or knee OA. The intervention did improve mental QoL, however, the intervention effect was not channelled via the selected mediators. The intervention did not cause a meaningful change in the hypothesised primary and alternative mediators, and these mediators were not associated with the selected outcomes.

Previous studies demonstrate that interventions have successfully improved weight, diet, physical activity and pain beliefs in patients with low back pain and knee OA. ${ }^{46-48}$ For example, Messier and colleagues report that a 6-month diet and exercise intervention led to a mean weight loss of $8.5 \mathrm{~kg}$ in participants with knee OA. ${ }^{48}$ However, most of these trials evaluated intensive face-to-face consultations and none were delivered using telephone health coaching. This difference in the mode of delivery might explain why our intervention did not exert an effect on the hypothesised mediators, whereas interventions in previous studies did. Although telephone interventions are effective in reducing weight and the behavioural determinants of weight (diet and physical activity) for the general population, ${ }^{49,50}$ their effectiveness for patients with chronic low back pain and knee OA have not been established. ${ }^{51,52}$ The telephone-based intervention used in our study was not effective in reducing self-reported weight, improving diet or physical activity, or changing erroneous pain beliefs in these patient groups.

Meta-analyses of observational cohort studies suggest that the hypothesised mediators are associated with patient outcomes. ${ }^{4-6,10,13,53}$ Although these meta-analyses report adjusted estimates, they did not consider the effects of unmeasured or residual confounding. ${ }^{54-56}$ Therefore, it is possible that these estimates were influenced by confounding bias. In our study, the ACME was stable across all possible levels of residual confounding, and we found no association between the majority of the hypothesised mediators and outcomes of pain intensity, disability, and QoL. However, in our study the absence of any association could be a result of lack of variability in the mediator scores, which may be a function of low variability in those measures in the sample at baseline and ineffectiveness of the intervention.

To our knowledge only one previous study of a lifestyle intervention in a similar population has undertaken causal mediation analyses. Foy et al. found that in adults with knee pain and diabetes who were overweight or obese, reduction in weight explained $98 \%$ of the intervention effect on disability. ${ }^{22}$ Conversely, we did not detect a mediating effect through 
weight loss. The difference in results may be because Foy et al. included patients with concomitant diabetes, which could have moderated the indirect effect. Furthermore, Foy et al. used an objective measure of weight, which may have increased the reliability and/or validity, compared to our self-reported measure. Lastly, Foy et al. did not undertake a sensitivity analysis to determine the impact of residual confounding on the mediator-outcome path, thus their estimate of the indirect effect through weight could be confounded.

Other studies suggest that improving lifestyle risk factors or changing pain beliefs positively affects patient outcomes in these patient groups. ${ }^{51,57,58}$ However, in the absence of causal mediation analyses, these studies can only assume that the intervention worked through hypothesised treatment targets. Without strong evidence for mediation through these targets, it remains possible that intervention acted via alternative mechanisms. Despite this uncertainty, trials without mediation analyses have informed clinical practice guidelines for chronic low back pain and knee OA. For example, for knee OA, weight loss is strongly recommended. ${ }^{14}$ Likewise, for chronic low back pain advice and education to correct erroneous pain beliefs is advised. ${ }^{15}$ Such guidelines should be better informed through robust evidence of treatment mechanisms. Collectively, the evidence to date does not convincingly demonstrate that overweight or obesity, poor diet, low levels of physical activity and erroneous pain beliefs are the appropriate mechanisms that should be targeted to improve pain intensity, disability, and QoL in patients with chronic low back pain or knee OA.

\section{Limitations}

The hypothesised mediators in this study were measured using self-reported questionnaires. Objective measures may increase the reliability and validity of the measurement of the hypothesised mediators. The mediators, diet and physical activity, were transformed from an ordinal and continuous scale respectively, to a binary scale to allow interpretation against the existing national guidelines. This may have reduced the responsiveness of these measures. We made three deviations from the published protocol. Although we transparently disclosed these deviations, they could have introduced bias.

\section{Implications for future research}

Although clinical guidelines advocate focusing on lifestyle risk factors and erroneous pain beliefs in patients with chronic low back pain or knee OA, there is uncertainty about whether they are causes of pain intensity, disability, and poor QoL. Future RCTs targeting lifestyle risk factors or erroneous pain beliefs in patients with chronic low back pain and knee OA should undertake mediation analyses to understand if the intervention changed the intended targets and if the targets were causally associated with the selected outcomes. To provide 
more convincing evidence, objective measures should be used when possible and sensitivity analyses assessing the effects of residual confounding should be undertaken.

\section{Clinical implications}

Our study found that the healthy lifestyle intervention delivered primarily using the telephone did not change the intended targets of weight, diet, physical activity and pain beliefs. Other studies suggest that a more intensive lifestyle intervention delivered face-to-face might change these targets. Currently, we cannot recommend that a lifestyle intervention delivered by telephone is preferable over face-to-face for patients with chronic low back pain and knee OA. As it remains unclear whether the hypothesised mediators in this study are causes of pain, disability and poor QoL in patients with chronic low back pain or knee OA, it is difficult to provide clinical guidance regarding prioritisation of these mediators. However, targeting these mediators, in particular, the lifestyle risk factors, may offer other health benefits such as improved cardiovascular disease risk, ${ }^{59}$ particularly for overweight or obese patients.

\section{Conclusions}

This study aimed to test the underlying causal mechanisms of a healthy lifestyle intervention for patients with chronic low back pain or knee OA who are overweight or obese. Our findings show that the intervention did not improve pain intensity, disability and physical QoL in participants with chronic low back pain and knee OA. The intervention did improve mental QoL, however, the intervention effect was not channelled via the selected mediators. The intervention did not cause a meaningful change in the hypothesised mediators, and these mediators were not associated with patient outcomes. 


\section{References}

1. GBD 2016 Disease and Injury Incidence and Prevalence Collaborators. Global, regional, and national incidence, prevalence, and years lived with disability for 328 diseases and injuries for 195 countries, 1990-2016: a systematic analysis for the Global Burden of Disease Study 2016. The Lancet. 2016;390(10100):1211-59.

2. Walker BF, Muller R, Grant WD. Low Back Pain in Australian Adults: The Economic Burden. Asia Pac J Public Health. 2003 Jul 1;15(2):79-87.

3. Access Economics (Firm) \& Diabetes Australia. The growing cost of obesity in 2008: three years on. Canberra: Diabetes Australia: Access Economics; 2008.

4. Shiri R, Karppinen J, Leino-Arjas P, Solovieva S, Viikari-Juntura E. The association between obesity and low back pain: a meta-analysis. Am J Epidemiol. 2010;171(2):135-54.

5. Leboeuf-Yde C. Body weight and low back pain. A systematic literature review of 56 journal articles reporting on 65 epidemiologic studies. Spine. 2000;25(2):226-37.

6. Blagojevic M, Jinks C, Jeffery A, Jordan KP. Risk factors for onset of osteoarthritis of the knee in older adults: a systematic review and meta-analysis. Osteoarthritis Cartilage. 2010 Jan;18(1):24-33.

7. Sowers MR, Karvonen-Gutierrez CA. The evolving role of obesity in knee osteoarthritis: Curr Opin Rheumatol. 2010 Sep;22(5):533-7.

8. Curioni CC, Lourenco PM. Long-term weight loss after diet and exercise: a systematic review. Int J Obes. 2005;29(10):1168.

9. Wu T, Gao X, Chen M, van Dam RM. Long-term effectiveness of diet-plus-exercise interventions vs. diet-only interventions for weight loss: a meta-analysis. Obes Rev. 2009 May;10(3):313-23.

10. Shiri R, Falah-Hassani K. Does leisure time physical activity protect against low back pain? Systematic review and meta-analysis of 36 prospective cohort studies. $\mathrm{Br} \mathrm{J}$ Sports Med. 2017 Jun 14;bjsports-2016-097352.

11. Lee J, Chang RW, Ehrlich-Jones L, Kwoh CK, Nevitt M, Semanik PA, et al. Sedentary behavior and physical function: Objective Evidence from the Osteoarthritis Initiative. Arthritis Care Res. 2015 Mar;67(3):366-73.

12. Rainville J, Smeets RJEM, Bendix T, Tveito TH, Poiraudeau S, Indahl AJ. Fearavoidance beliefs and pain avoidance in low back pain-translating research into clinical practice. Spine J. 2011 Sep 1;11(9):895-903.

13. Wertli MM, Rasmussen-Barr E, Weiser S, Bachmann LM, Brunner F. The role of fear avoidance beliefs as a prognostic factor for outcome in patients with nonspecific low back pain: a systematic review. Spine J. 2014 May 1;14(5):816-836.e4.

14. National Clinical Guideline Centre (NICE). Osteoarthritis: Care and management in adults. NICE Clinical Guideline 177. London, UK: NICE; 2014. 
15. National Clinical Guideline Centre (NICE). Low Back Pain and Sciatica in Over 16s: Assessment and Management. NICE Clinical Guidelines NG59. London, UK: NICE; 2016.

16. Williams A, Wiggers J, O'Brien KM, Wolfenden L, Yoong SL, Hodder RK, et al. The effectiveness of a healthy lifestyle intervention, for chronic low back pain: a randomised controlled trial. PAIN. 2018;doi: 10.1097/j.pain.0000000000001198. [In Press].

17. O'Brien KM, Wiggers J, Williams A, Campbell E, Hodder RK, Wolfenden L, et al. Telephone-based weight loss support for patients with knee osteoarthritis: a pragmatic randomized controlled trial. Osteoarthritis Cartilage. 2018;doi: 10.1016/j.joca.2018.01.003. [In Press].

18. Lee H, Mansell G, McAuley JH, Kamper SJ, Hübscher M, Moseley GL, et al. Causal mechanisms in the clinical course and treatment of back pain. Best Pract Res Clin Rheumatol. 2016;30(6):1074-83.

19. Mansell G, Hill JC, Kamper SJ, Kent P, Main C, van der Windt DA. How Can We Design Low Back Pain Intervention Studies to Better Explain the Effects of Treatment? Spine. 2014 Mar 1;39(5):E305.

20. Imai K, Keele L, Tingley D. A general approach to causal mediation analysis. Psychol Methods. 2010;15(4):309-34.

21. Lee H, Lamb S. Advancing physical therapist interventions by investigating causal mechanisms. Phys Ther. 2017;97(12):1119-21.

22. Foy CG, Lewis CE, Hairston KG, Miller GD, Lang W, Jakicic JM, et al. Intensive Lifestyle Intervention Improves Physical Function Among Obese Adults With Knee Pain: Findings From the Look AHEAD Trial. Obesity. 2011 Jan 1;19(1):83-93.

23. Williams A, Wiggers J, O'Brien KM, Wolfenden L, Yoong S, Campbell E, et al. A randomised controlled trial of a lifestyle behavioural intervention for patients with low back pain, who are overweight or obese: study protocol. BMC Musculoskelet Disord. 2016;17(1):1.

24. O'Brien KM, Wiggers J, Williams A, Campbell E, Wolfenden L, Yoong S, et al. Randomised controlled trial of referral to a telephone-based weight management and healthy lifestyle programme for patients with knee osteoarthritis who are overweight or obese: a study protocol. BMJ Open. 2016;6(3):e010203.

25. Lee H, Wiggers J, Kamper SJ, Williams A, O'Brien KM, Hodder RK, et al. Mechanism evaluation of a lifestyle intervention for patients with musculoskeletal pain who are overweight or obese: protocol for a causal mediation analysis. BMJ Open. 2017 Jun;7(6):e014652.

26. O'Hara BJ, Phongsavan P, Venugopal K, Eakin EG, Eggins D, Caterson H, et al. Effectiveness of Australia's Get Healthy Information and Coaching Service: translational research with population wide impact. Prev Med. 2012 Oct;55(4):292-8.

27. Ryan RM, Deci EL. Self-determination theory and the facilitation of intrinsic motivation, social development, and well-being. Am Psychol. 2000;55(1):68-78. 
28. Australian Institute of Health and Welfare (AlHW). The Active Australia Survey: a guide and manual for implementation, analysis and reporting. Canberra, Australia: AlHW; 2003.

29. Brown W, Trost S, Bauman A, Mummery K, Owen N. Test-retest reliability of four physical activity measures used in population surveys. J Sci Med Sport. 2004;7(2):20515.

30. Booth M, Bauman AE, Timperio A, Salmon J, Trost S. Measurement of adult physical activity: reliability, comparison and validity of self-report surveys for population surveillance. Summary and recommendations. 2002.

31. Centre for Epidemiology and Research NSW population health survey. Sydney; 2014.

32. CATI Technical Reference Group. Population Health Monitoring and Surveillance: Question Development Field Testing. 2004.

33. Flood VM, Wen LM, Hardy LL, Rissel C, Simpson JM, Baur LA. Reliability and validity of a short FFQ for assessing the dietary habits of 2-5-year-old children, Sydney, Australia. Public Health Nutr. 2014;17(3):498-509.

34. Jensen MP, Karoly P, Huger R. The development and preliminary validation of an instrument to assess patients' attitudes toward pain. J Psychosom Res. 1987;31(3):393-400.

35. Jensen MP, Keefe FJ, Lefebvre JC, Romano JM, Turner JA. One-and two-item measures of pain beliefs and coping strategies. Pain. 2003;104(3):453-69.

36. Hawker GA, Mian S, Kendzerska T, French M. Measures of adult pain: Visual Analog Scale for Pain (VAS Pain), Numeric Rating Scale for Pain (NRS Pain), McGill Pain Questionnaire (MPQ), Short-Form McGill Pain Questionnaire (SF-MPQ), Chronic Pain Grade Scale (CPGS), Short Form-36 Bodily Pain Scale (SF-36 BPS), and Measure of Intermittent and Constant Osteoarthritis Pain (ICOAP). Arthritis Care Res. 2011;63(November):240-52.

37. Roland M, Morris R. A study of the natural history of back pain: part I: development of a reliable and sensitive measure of disability in low-back pain. Spine. 1983;8(2):141-4.

38. Bellamy N. WOMAC user guide IX. Brisbane: Nicholas Bellamy; 2009.

39. Ware J, Kosinski M, Bjorner J, Turner-Bowker D, Gandek B, Maruish M. User's Manual for the SF-12v2 Health Survey (with a Supplement Documenting SF-12 Health Survey). Boston, MA: Lincoln, RI: QualityMetric Incorporated; 2002.

40. Jensen MP, Turner JA, Romano JM, Fisher LD. Comparative reliability and validity of chronic pain intensity measures. PAIN. 1999 Nov 1;83(2):157-62.

41. Tingley D, Yamamoto T, Hirose K, Keele L, Imai K. mediation: R Package for Causal Mediation Analysis. J Stat Softw. 2014;59(5):1-38.

42. Landau S, Emsley R, Dunn G. Beyond total treatment effects in RCTs: why we need to measure outcomes at baseline when investigating mediation. Trials. 2015;16(Suppl 2):O42. 
43. National Health and Medical Research Council (NHMRC). Australian Dietary Guidelines. Canberra: NHMRC; 2013.

44. Brown W, Bauman A, Bull F, Burton N. Development of Evidence-Based Physical Activity Recommendations for Adults (18 - 64 years). Report prepared for the Australian Government Department of Health; 2012.

45. Van Cleave JH, Egleston BL, Bourbonniere M, McCorkle R. Combining Extant Datasets with Differing Outcome Measures Across Studies of Older Adults After Cancer Surgery. Res Gerontol Nurs. 2011 Jan 1;4(1):36-46.

46. Ben-Ami N, Chodick G, Mirovsky Y, Pincus T, Shapiro Y. Increasing Recreational Physical Activity in Patients With Chronic Low Back Pain: A Pragmatic Controlled Clinical Trial. J Orthop Sports Phys Ther. 2017 Feb;47(2):57-66.

47. Moseley GL. Evidence for a direct relationship between cognitive and physical change during an education intervention in people with chronic low back pain. Eur J Pain. 2004 Feb 1;8(1):39-45.

48. Messier SP, Loeser RF, Mitchell MN, Valle G, Morgan TP, Rejeski WJ, et al. Exercise and Weight Loss in Obese Older Adults with Knee Osteoarthritis: A Preliminary Study. J Am Geriatr Soc. 2000 Sep 1;48(9):1062-72.

49. Appel LJ, Clark JM, Yeh H-C, Wang N-Y, Coughlin JW, Daumit G, et al. Comparative effectiveness of weight-loss interventions in clinical practice. N Engl J Med. 2011;365(21):1959-68.

50. Goode AD, Reeves MM, Eakin EG. Telephone-delivered interventions for physical activity and dietary behavior change: an updated systematic review. Am J Prev Med. 2012;42(1):81-8.

51. Christensen R, Bartels EM, Astrup A, Bliddal H. Effect of weight reduction in obese patients diagnosed with knee osteoarthritis: a systematic review and meta-analysis. Ann Rheum Dis. 2007;66(4):433-9.

52. Wai EK, Rodriguez S, Dagenais S, Hall H. Evidence-informed management of chronic low back pain with physical activity, smoking cessation, and weight loss. Spine J Off J North Am Spine Soc. 2008;8(1):195-202.

53. Wertli MM, Burgstaller JM, Weiser S, Steurer J, Kofmehl R, Held U. Influence of Catastrophizing on Treatment Outcome in Patients With Nonspecific Low Back Pain: A Systematic Review. Spine. 2014 Feb 1;39(3):263.

54. VanderWeele TJ. Unmeasured confounding and hazard scales: sensitivity analysis for total, direct, and indirect effects. Eur J Epidemiol. 2013 Feb;28(2):113-7.

55. VanderWeele TJ, Ding P. Sensitivity Analysis in Observational Research: Introducing the E-Value. Ann Intern Med. 2017 Aug 15;167(4):268-74.

56. Rosenbaum PR. Discussing Hidden Bias in Observational Studies. Ann Intern Med. 1991 Dec 1;115(11):901.

57. Somers TJ, Blumenthal JA, Guilak F, Kraus VB, Schmitt DO, Babyak MA, et al. Pain coping skills training and lifestyle behavioral weight management in patients with knee osteoarthritis: A randomized controlled study. PAIN. 2012 Jun;153(6):1199-209. 
58. Burton AK, Waddell G, Tillotson KM, Summerton N. Information and Advice to Patients With Back Pain Can Have a Positive Effect: A Randomized Controlled Trial of a Novel Educational Booklet in Primary Care. Spine. 1999;24:2484.

59. Li J, Siegrist J. Physical Activity and Risk of Cardiovascular Disease-A Meta-Analysis of Prospective Cohort Studies. Int J Environ Res Public Health. 2012 Jan 26;9(12):391-407. 